\title{
Factors predicting the occurrence of net adverse clinical and cerebral events in patients with acute coronary syndrome treated with clopidogrel or ticagrelor in combination with aspirin: a real- world study
}

\author{
Man Huang ${ }^{1 \#}$, Dandan $\mathrm{Li}^{2 \#}$, Lanting $\mathrm{Li}^{3}$, Yan Wang ${ }^{1}$, Linlin Zhang ${ }^{4}$, Yundai Chen ${ }^{2 *}$, Dao Wen Wang ${ }^{1 *}$ \\ ${ }^{1}$ Division of Cardiology, Department of Internal Medicine, Tongji Hospital, Tongji Medical College, Huazhong University of Science and \\ Technology, Wuhan, China; ${ }^{2}$ Senior Department of Cardiology, the Sixth Medical Center of PLA General Hospital, Beijing, China; ${ }^{3}$ Shanghai Palan \\ DataRx Co., Ltd., Shanghai, China; ${ }^{4}$ Medical Affair, Sanofi (China) Investment, Co., Ltd., Beijing, China \\ Contributions: (I) Conception and design: M Huang, D Li, Y Chen, DW Wang; (II) Administrative support: Y Wang; (III) Provision of study materials \\ or patients: Y Chen, DW Wang; (IV) Collection and assembly of data: M Huang, D Li; (V) Data analysis and interpretation: L Li, M Huang, D Li, \\ L Zhang, Y Chen, DW Wang; (VI) Manuscript writing: All authors; (VII) Final approval of manuscript: All authors. \\ \#These authors contributed equally to this work and should be considered as co-first authors. \\ *These authors contributed equally to this work as co-corresponding authors. \\ Correspondence to: Dao Wen Wang. Division of Cardiology, Department of Internal Medicine, Tongji Hospital, Tongji Medical College, Huazhong \\ University of Science and Technology, Wuhan 430030, China. Email: dwwang@tjh.tjmu.edu.cn; Yundai Chen. Senior Department of Cardiology, the \\ Sixth Medical Center of PLA General Hospital, Beijing, China. Email: cyundai@vip.163.com.
}

Background: This study aimed to establish the factors influencing the clinical benefits of ticagrelor and clopidogrel for acute coronary syndrome (ACS) patients undergoing percutaneous coronary intervention (PCI) surgery.

Methods: A multicenter, retrospective, real-world study was conducted on patients with ACS whose data were sourced from 3 databases, namely the BRIC-ACS(I) study, COSTIC study, and 301 Hospital PCI patient database from January 2014 to October 2017. The primary endpoint was net adverse clinical and cerebral events (NACCE).

Results: A total of 7,236 ACS patients were included, of which 4,444 patients (61.4\%) and 2,792 patients $(38.6 \%)$ were in the clopidogrel dual antiplatelet therapy (DAPT) group and ticagrelor DAPT group, respectively. The hazard ratio (HR) for NACCE was significantly higher in patients aged $\geq 65$ years than those aged $\leq 65$ years in the clopidogrel DAPT group (HR: 2.15, 95\% CI: 1.68-2.76) and ticagrelor DAPT group (HR: 1.75, 95\% CI: 1.34-2.29). In patients treated with clopidogrel DAPT, patients with unstable angina had a significantly lower HR for NACCE than patients with ST-elevation myocardial infarction. Use of beta blockers (HR: 0.77, 95\% CI: 0.60-0.99) was an influencing factor in patients treated with clopidogrel DAPT, whereas in patients treated with ticagrelor DAPT, only smoking status (HR: 0.75, 95\% CI: 0.57-0.99) was a significant influencing factor.

Conclusions: Age, hypertension status, and presence or absence of unstable angina were factors influencing the composite outcome of NACCE. The selection of patients to be treated with either clopidogrel DAPT or ticagrelor DAPT depending on the presence or absence of factors influencing treatment outcome may improve therapeutic management.

Keywords: Aspirin; clopidogrel; dual antiplatelet therapy (DAPT); net adverse clinical and cerebral events (NACCE); ticagrelor

Submitted Dec 17, 2021. Accepted for publication Jan 20, 2022.

doi: $10.21037 / \mathrm{atm}-21-7038$

View this article at: https://dx.doi.org/10.21037/atm-21-7038 


\section{Introduction}

In recent years, China has faced an epidemic of acute coronary syndrome (ACS), which results in a high rate of mortality and morbidity $(1,2)$. The surge in the incidence of risk factors for ACS with age remains a grave concern in mainland China. Moreover, due to the drastic rise in the number of patients with ACS, the percentage of percutaneous coronary interventions (PCIs) performed in China has also increased over the years. According to the current guidelines from various heart associations around the world, the use of ticagrelor is recommended over clopidogrel as it reduces the occurrence of major cardiovascular events (MACE) including myocardial infarction (MI) in patients with ACS (2). These recommendations are in accordance with the results of the Platelet Inhibition and Patient Outcomes (PLATO) trial conducted in 2009 (3).

The activation of platelets after PCI in ACS patients is critical in the development of thrombosis. Therefore, to prevent atherothrombotic events, the use of antiplatelet agents is encouraged (4). Dual antiplatelet therapy (DAPT) employing a combination of clopidogrel (P2Y12 inhibitor) and aspirin is recommended for the prevention of recurrent thrombotic events in patients with ACS during PCI. However, the pharmacological limitations associated with clopidogrel DAPT have led to the development of novel reversible P2Y12 receptor antagonists (5). According to the current guidelines based on previously conducted clinical trials [PLATO trial (3)], the use of ticagrelor as a novel potent $\mathrm{P} 2 \mathrm{Y} 12$ inhibitor is recommended after PCI for 12 months to reduce coronary thrombosis and mortality in patients with ACS (6). Studies have shown that DAPT reduces the risk of stent-related and spontaneous recurrent ischemic events in patients with ACS undergoing PCI. Nevertheless, DAPT (combination of ticagrelor and aspirin), despite being effective in reducing thrombotic complications, is associated with an increased the risk of bleeding events. The bleeding risk associated with them was solely considered as an inevitable and acceptable complication. Some new agents have modified the balance between the absolute risk reduction in ischemic events and the absolute risk increase in bleeding events (7). Therefore, to consider DAPT it is essential to assess the risks that be able to balance the occurrence of thrombotic versus hemorrhagic events.

Previous studies have questioned either the efficacy of DAPT involving ticagrelor and clopidogrel or the risk of thrombotic and hemorrhagic events associated with these agents (8). Clinical trial data of patients from Europe and America have been contradictory to those of East Asian patients regarding to the efficacy and bleeding events occurrence of ticagrelor compared with clopidogrel (9). Furthermore, specific patient baseline and clinical factors could lead to the differential clinical outcomes in patients with ACS treated with ticagrelor DAPT or clopidogrel after PCI. Therefore, identification of such influencing factors helps in the stratification of patients for clinical interventions and better clinical outcomes. However, there is no evidence on factors predicting the occurrence of net adverse clinical and cerebral events (NACCE) in Chinese patients with ACS treated with clopidogrel DAPT and ticagrelor DAPT. Therefore, in the current study, the factors influencing the composite outcome of NACCE in patients treated with clopidogrel DAPT and ticagrelor DAPT were analyzed in real-world clinical settings.

We present the following article in accordance with the STROBE reporting checklist (available at https://atm. amegroups.com/article/view/10.21037/atm-21-7038/rc).

\section{Methods}

\section{Study design and population}

This is a nationwide, multicenter, retrospective, noninterventional, real-world study. The database included 31 centers with 7,862 Chinese adult ACS patients who underwent PCI, and these patients were discharged from hospital between January 2014 and October 2017. Patient data were obtained from the BRIC-ACS(I) study (2,381 patients) (6), COSTIC study (4,447 patients) (10), and cases from the Sixth Medical Center of PLA General Hospital PCI patient database (1,034 patients). The data for the patients who underwent PCI with second- or thirdgeneration drug-eluting stent (DES) implantation, and were treated either with clopidogrel or ticagrelor plus aspirin for 12 months of follow-up were included in this real-world study. The patients were excluded from this study if the criteria below meet: (I) patients were treated with anticoagulants or drugs could increase the risk of bradycardia; (II) patients received treatment switched between multiple P2Y12 inhibitors; (III) no aspirin prescription; (IV) patients with malignancies. This study was approved by the institutional review board of the Sixth Medical Center of PLA General Hospital (approval number: S2020-253-01). The study was conducted in accordance with the Declaration of Helsinki (as revised in 
2013). In addition, informed consent was obtained from the participants involved.

\section{Outcomes and measures}

The primary outcome measure of the study was the incidence of NACCE, including all-cause death, non-fatal stroke, nonfatal MI, and bleeding as per Bleeding Academic Research Consortium (BARC) type $\geq 2$ (excluding BARC type 4) bleeding within 12 months after discharge (11). The patientrelated factors influencing the occurrence of NACCE were analyzed.

Type 4a MI was defined as the elevation in cardiac troponin values (cTn) to $>99 \%$ of the upper reference limit and at least one of the following: symptoms of myocardial ischemia; ischemic electrocardiographic (ECG) changes; development of pathological Q waves; imaging evidence of loss of viable myocardium or regional wall motion abnormality that was presumed to be in a pattern, consistent with an ischemic etiology (12). Stroke was defined as focal loss of neurological function lasting at least 24 hours, regardless of whether the symptom was caused by a hemorrhagic or ischemic event (13).

A bleeding complication was defined as any bleeding event recorded by the treating cardiologist. BARC type 2 bleeding was defined as any evident, actionable indication of hemorrhage (e.g., more bleeding than would be expected for a clinical circumstance, including bleeding found by imaging alone) that did not fit the criteria for type 3 , 4 , or 5 but required nonsurgical medical intervention by a healthcare professional, increased level of care, hospitalization, or prompted evaluation (14).

\section{Statistical analysis}

Patients with 12 months of complete follow-up data were considered for statistical analysis. Continuous variables were presented as mean \pm standard deviation, and categorical variables were presented as number with percentage. For the difference comparison, Student's unpaired $t$-test was performed for continuous variables, Chi-square test was used for categorical variables. Fisher's exact test was used when the expected frequency less than 1 or $20 \%$ had expected frequency of equal to or less than 5. The BRICACS score was calculated for each patient enrolled in the current study. The prediction of BARC type $\geq 2$ (excluding BARC type 4) bleeding within 1-year post discharge was determined based on different thresholds of the score and compared with the true bleeding outcome. For influential factors of NACCE in patients within 1 year after discharge, Akaike's information criterion was used as the stopping rule for defaults, and Cox regression analysis (backward stepwise variable selection method) was performed. The hazard ratio (HR) and 95\% confidence interval (CI) were calculated for characteristics with $\mathrm{P}<0.05$. Statistical analyses were performed using $\mathrm{R}$ (version 3.5.1).

\section{Results}

A total of 7,236 Chinese patients (aged $\geq 18$ years) with ACS who underwent PCI from January 2014 and October 2017 were enrolled. The patients were included from the BRICACS(I) study (2,012 patients), the COSTIC study (4,434 patients), and the 301 Hospital PCI patient database (790 patients).

A total of 4,444 patients (61.5\%) were received clopidogrel DAPT treatment at discharge while the remaining 2,792 (38.5\%) were treated with ticagrelor DAPT.

\section{Baseline and procedural characteristics}

The summary for all patients' baseline characteristics are listed in Table 1. Patients showed younger age in the group treated with ticagrelor $(59.53 \pm 10.19$ years $)$ compared with the group treated with clopidogrel and aspirin (61.11 \pm 10.68 years). The ticagrelor DAPT group comprised more males $(76.0 \%)$ than the clopidogrel DAPT group (72.4\%, $\mathrm{P}<0.05)$ and had a lower burden of peripheral arterial disease [ticagrelor DAPT vs. clopidogrel DAPT: 356 (12.8\%) vs. 466 (10.5\%)]. Patients treated with clopidogrel and aspirin reported a higher percentage of cerebrovascular disease $(7.1 \%)$ than those in the ticagrelor and aspirin group $(5.7 \%)$. The most frequent clinical presentation was unstable angina for both the clopidogrel and aspirin group $(59.6 \%)$ and the ticagrelor and aspirin group (60.2\%). Transradial access was the preferred approach for PCI in patients treated with clopidogrel and aspirin (91.5\%) and ticagrelor and aspirin (93.8\%). The incidence of multivessel disease was higher in patients treated with ticagrelor and aspirin $(22.0 \%)$ compared to that in the clopidogrel and aspirin group $(10.5 \%, \mathrm{P}<0.05)$.

\section{Influential factors for NACCE in the clopidogrel DAPT and ticagrelor DAPT population}

The results of the multivariate Cox regression analysis 
Table 1 Baseline characteristics by antiplatelet treatment group: clopidogrel DAPT vs. ticagrelor DAPT

\begin{tabular}{|c|c|c|c|c|}
\hline Characteristics & Total & Clopidogrel + aspirin & Ticagrelor + aspirin & ASD \\
\hline \multicolumn{5}{|l|}{ Demographic characteristics } \\
\hline Male, n (\%) & $5,342(73.8)$ & $3,219(72.4)$ & $2,123(76.0)$ & 0.08 \\
\hline Age, mean $\pm S D, y$ & $60.5 \pm 10.52$ & $61.11 \pm 10.68$ & $59.53 \pm 10.19$ & 0.15 \\
\hline Cerebrovascular disease & $473(6.5)$ & $313(7.1)$ & $160(5.7)$ & 0.05 \\
\hline Peptic ulcer & $193(2.7)$ & $139(3.1)$ & $54(2.0)$ & 0.08 \\
\hline Hypertension & $4,347(60.1)$ & $2,669(60.1)$ & $1,678(60.1)$ & $<0.01$ \\
\hline Prior bleeding & $86(1.2)$ & $60(1.4)$ & $26(1.0)$ & 0.04 \\
\hline \multicolumn{5}{|l|}{ Clinical presentation, n (\%) } \\
\hline STEMI & $1,753(24.2)$ & $1,087(24.5)$ & $666(23.9)$ & 0.01 \\
\hline NSTEMI & $1,151(15.9)$ & 707 (15.9) & $444(15.9)$ & $<0.01$ \\
\hline UA & 4,331 (59.9) & $2,649(59.6)$ & 1,682 (60.2) & 0.01 \\
\hline \multicolumn{5}{|c|}{ Lesion and procedure characteristics, n (\%) } \\
\hline Three vessel disease & $1,074(14.9)$ & $465(10.5)$ & $609(22.0)$ & 0.31 \\
\hline Transradial & $6,686(92.4)$ & $4,068(91.5)$ & $2,618(93.8)$ & 0.09 \\
\hline
\end{tabular}

DAPT, dual antiplatelet therapy; COPD, chronic obstructive pulmonary disease; DES, drug-eluting stent; MI, myocardial infarction; UA, unstable angina; STEMI, ST-elevation myocardial infarction; NSTEMI, non-ST-elevation myocardial infarction; ASD, absolute standardized difference.

with NACCE as the outcome are presented in Table 2. The most significant factors in the population receiving both clopidogrel DAPT and ticagrelor DAPT were age, treatment regimen with ticagrelor DAPT, and presence or absence of unstable angina $(\mathrm{P}<0.001)$. As shown in Table 3, patients aged $\geq 65$ years experienced a higher incidence of NACCE (HR: 2.03, 95\% CI: 1.70-2.44) than those aged $<65$ years. Patients with a history of hypertension in both treatment groups experienced increased NACCE (HR: 1.21, 95\% CI: 1.01-1.45) whereas patients with unstable angina had a low incidence of NACCE (HR: 0.66, 95\%
CI: 0.54-0.82). Among the different clinical presentations, the HR associated with unstable angina was significantly lower (HR: 0.66, 95\% CI: 0.54-0.82) than either non-STelevation myocardial infarction (NSTEMI) or ST-elevation myocardial infarction (STEMI).

\section{Influential factors for NACCE in the clopidogrel DAPT population}

For the population receiving only clopidogrel plus aspirin, the most significant prognostic factors included age, 
Table 2 Influential factors for NACCE in the clopidogrel DAPT and ticagrelor DAPT population

\begin{tabular}{lcc}
\hline Factors & HR $(95 \%$ Cl $)$ & P value \\
\hline Age & Ref & - \\
$<65$ y & $2.03(1.70,2.44)$ & $<0.001$ \\
$\geq 65$ y & Ref & - \\
Antiplatelet therapy & $1.48(1.24,1.77)$ & $<0.001$ \\
Clopidogrel and aspirin & $1.24(1.03,1.50)$ & 0.025 \\
Ticagrelor and aspirin & $1.32(0.97,1.78)$ & 0.076 \\
Hypertension & & \\
Cerebrovascular disease & Ref & - \\
Clinical presentation & $1.11(0.86,1.43)$ & 0.430 \\
STEMI & $0.66(0.54,0.82)$ & $<0.001$ \\
NSTEMI & $0.78(0.65,0.94)$ & 0.010 \\
\hline UA & &
\end{tabular}

NACCE, net adverse clinical and cerebral events; DAPT, dual antiplatelet therapy; UA, unstable angina; STEMI, ST-elevation myocardial infarction; NSTEMI, non-ST-elevation myocardial infarction; HR, hazard ratio.

Table 3 Influential factors for NACCE in the clopidogrel DAPT population

\begin{tabular}{lcc}
\hline Factors & HR $(95 \% \mathrm{Cl})$ & P value \\
\hline Age & Ref & - \\
$<65 \mathrm{y}$ & $2.15(1.68,2.76)$ & $<0.001$ \\
$\geq 65 \mathrm{y}$ & $1.24(0.96,1.60)$ & 0.105 \\
Hypertension & $1.40(0.95,2.07)$ & 0.088 \\
Cerebrovascular disease & Ref & - \\
Clinical presentation & $1.13(0.80,1.59)$ & 0.476 \\
STEMI & $0.66(0.49,0.89)$ & 0.006 \\
NSTEMI & $0.77(0.60,0.99)$ & 0.044 \\
UA & & \\
Beta blocker & Ref & - \\
Data source & $0.92(0.71,1.18)$ & 0.516 \\
BRIC-ACS(I) & $0.40(0.16,1.00)$ & 0.049 \\
COSTIC & & \\
301 Hospital & &
\end{tabular}

NACCE, net adverse clinical and cerebral events; DAPT, dual antiplatelet therapy; UA, unstable angina; STEMI, ST-elevation myocardial infarction; NSTEMI, non-ST-elevation myocardial infarction; HR, hazard ratio.
Table 4 Influential factors for NACCE in the ticagrelor DAPT population

\begin{tabular}{lcc}
\hline Factors & HR $(95 \% \mathrm{Cl})$ & P value \\
\hline Age & Ref & - \\
$<65 \mathrm{y}$ & $1.75(1.34,2.29)$ & $<0.001$ \\
$\geq 65 \mathrm{y}$ & $0.75(0.57,0.99)$ & 0.041 \\
Smoker & $1.30(0.97,1.74)$ & 0.076 \\
Number of DESs $\geq 2$ & $0.79(0.59,1.04)$ & 0.094 \\
Beta blocker &
\end{tabular}

NACCE, net adverse clinical and cerebral events; DAPT, dual antiplatelet therapy; HR, hazard ratio; DES, drug-eluting stent.

presence or absence of unstable angina, treatment with beta blockers, and data source from the 301 Hospital $(\mathrm{P}<0.05)$. The HR was significantly higher (HR: 2.15, 95\% CI: 1.68 2.76 ) in patients aged $\geq 65$ years than those aged $<65$ years (Table 3). Patients with unstable angina experienced a lower incidence of NACCE than patients without unstable angina (HR: $0.66,95 \%$ CI: 0.49-0.89) and patients treated at the 301 Hospital experienced a higher incidence of NACCE than those treated at other centers (HR: 0.40, 95\% CI: 0.16-1.00) with the clopidogrel DAPT regimen.

\section{Influential factors for NACCE in the ticagrelor DAPT population}

The significant influential factors associated with the population receiving ticagrelor DAPT were age and smoking status $(\mathrm{P}<0.05)$, as shown in Table 4. Patients aged $\geq 65$ years were more prone to NACCE (HR: 1.75, 95\% CI: $1.34-2.29)$ compared to those aged $<65$ years. Patients with cardiovascular risk factors (smoking) showed a lower incidence of NACCE (HR: 0.75, 95\% CI: 0.57-0.99).

\section{Validation of the BRIC-ACS score}

Among the 5,481 patients from the COSTIC study and 301 Hospital, 806 patients had all the data required for calculating the score. The area under curve (AUC) was 0.502 when the median BRIC-ACS score (32.1) of the population was set as the threshold for predicting the occurrence of BARC type $\geq 2$ post-discharge bleeding (PDB). The sensitivity, specificity, positive predictive value, and negative predictive value were $0.533,0.501,0.040$, and 0.965 , respectively. 


\section{Discussion}

For patients with ACS, understanding the factors influencing the incidence of NACCE is significant due to its implication in selecting appropriate patients for treatment with clopidogrel DAPT or ticagrelor DAPT. In the present retrospective cohort study, the performance of the BRICACS score among Chinese ACS patients undergoing PCI was validated, and different factors influencing the occurrence of NACCE in patients with ACS treated with either clopidogrel DAPT or ticagrelor DAPT within 1-year post PCI in mainland China were reported.

The influential factors for NACCE in the total population receiving clopidogrel DAPT or ticagrelor DAPT were age, ticagrelor DAPT regimen, hypertension status, presence or absence of unstable angina, and treatment with beta blockers. Among the patients of the two different age groups, elderly patients ( $\geq 65$ years of age) being treated for ACS were more prone to NACCE. Several previous studies demonstrated that elderly patients with ACS are at higher risk of recurrent NACCE compared to younger patients $(2,15,16)$. Previously, Bian et al. conducted a study to evaluate the optimal antiplatelet therapy for Chinese patients with coronary artery disease (OPT-CAD) which showed a lower incidence of net adverse clinical events in the DAPT group than the single antiplatelet therapy group (17). Furthermore, subgroup analysis based on the Global Registration of Acute Coronary Events (GRACE) score revealed that the net adverse clinical outcomes of continued DAPT were not significantly different in terms of age [relative risk (RR) (95\% CI): $\geq 65: 0.54$ (0.33-0.88), <65: 0.75 (0.29-1.92), P for interaction $=0.54](17)$. However, this disparity might be due to the difference in scoring system adopted among these studies or due to other baseline differences among the patients included in the study. Furthermore, influential factors for NACCE in the clopidogrel DAPT population was age, presence or absence of unstable angina, treatment with beta blockers, and data source from the 301 Hospital $(\mathrm{P}<0.05)$.

The influential factors for NACCE in the ticagrelor DAPT population were age and smoking status $(\mathrm{P}<0.05)$. Hypertension is a known influential factor for the development of ACS (18). In agreement with earlier reports, results from the current study confirmed that hypertension was associated with an increased risk of NACCE, which was significantly higher in the ticagrelor DAPT group compared with the clopidogrel DAPT group during the 1-year follow-up.

The primary function of DAPT is assessed by platelet aggregation. Although our study reported age, smoking status, and ST segment elevation as significant factors predicting efficacy in patients treated with ticagrelor and clopidogrel, a previous study estimating the platelet aggregation effect of clopidogrel and ticagrelor reported contradictory findings. A previous study by Tatarunas et al. reported no significant difference in platelet aggregation in patients treated with ticagrelor and clopidogrel stratified by age $(<65$ and $\geq 65$ ), smoking status, and gender. The study also reported significant differences in patients treated with clopidogrel stratified by ST segment elevation (19). Our current study also reported ST segment elevation to be a significant factor in predicting NACCE in patients treated with clopidogrel. This suggests that the observed difference in NACCE in patients treated with clopidogrel stratified by ST segment elevation could be due to the difference in platelet aggregation in the respective groups. In other stratified groups, the observed clinical effect was not substantiated about the difference in platelet aggregation. This suggests that the underlying mechanisms of the observed associations between treatment groups and clinical/demographic factors should be elucidated in further clinical studies.

Our study has certain limitations. Despite including a considerable number of patients, the patients were not randomized, and therefore selection bias may have existed. Furthermore, owing to the retrospective nature of data acquisition, the data relating to post-discharge changes in the DAPT regimen were not available. Nevertheless, each patient was recommended to continue DAPT for a minimum of 12 months after discharge, in keeping with the current guidelines.

\section{Conclusions}

The analysis of factors influencing the occurrence of NACCE in patients with ACS undergoing PCI showed that age, hypertension status, and presence or absence of unstable angina were influential factors on the composite outcome of NACCE. The BRIC-ACS risk score was confirmed as a useful tool for PDB occurrence in patients with ACS. The selection of patients to be treated with either clopidogrel DAPT or ticagrelor DAPT depending on the presence or absence of the factors influencing treatment outcome may improve the therapeutic management of patients with ACS undergoing PCI. 


\section{Acknowledgments}

Funding: This study was funded by Sanofi.

\section{Footnote}

Reporting Checklist: The authors have completed the STROBE reporting checklist. Available at https://atm. amegroups.com/article/view/10.21037/atm-21-7038/rc

Data Sharing Statement: Available at https://atm.amegroups. com/article/view/10.21037/atm-21-7038/dss

Conflicts of Interest: All authors have completed the ICMJE uniform disclosure form (available at https://atm. amegroups.com/article/view/10.21037/atm-21-7038/coif). All authors report that this study was funded by Sanofi. Dr. LL is from Shanghai Palan DataRx Co., Ltd. Dr. LZ is from Medical Affair, Sanofi (China) Investment, Co., Ltd. The authors have no other conflicts of interest to declare.

Ethical Statement: The authors are accountable for all aspects of the work in ensuring that questions related to the accuracy or integrity of any part of the work are appropriately investigated and resolved. This study was approved by the institutional review board of the Sixth Medical Center of PLA General Hospital (approval number: S2020-253-01). The study was conducted in accordance with the Declaration of Helsinki (as revised in 2013). In addition, informed consent was obtained from the participants involved.

Open Access Statement: This is an Open Access article distributed in accordance with the Creative Commons Attribution-NonCommercial-NoDerivs 4.0 International License (CC BY-NC-ND 4.0), which permits the noncommercial replication and distribution of the article with the strict proviso that no changes or edits are made and the original work is properly cited (including links to both the formal publication through the relevant DOI and the license). See: https://creativecommons.org/licenses/by-nc-nd/4.0/.

\section{References}

1. Ma LY, Chen WW, Gao RL, et al. China cardiovascular diseases report 2018: an updated summary. J Geriatr Cardiol 2020;17:1-8.

2. You SC, Rho Y, Bikdeli B, et al. Association of
Ticagrelor vs Clopidogrel With Net Adverse Clinical Events in Patients With Acute Coronary Syndrome Undergoing Percutaneous Coronary Intervention. JAMA 2020;324:1640-50.

3. Wallentin L, Becker RC, Budaj A, et al. Ticagrelor versus clopidogrel in patients with acute coronary syndromes. $\mathrm{N}$ Engl J Med 2009;361:1045-57.

4. Xue $\mathrm{Y}, \mathrm{Hu} Z$, Jing $\mathrm{Y}$, et al. Efficacy assessment of ticagrelor versus clopidogrel in Chinese patients with acute coronary syndrome undergoing percutaneous coronary intervention by data mining and machine-learning decision tree approaches. J Clin Pharm Ther 2020;45:1076-86.

5. Milluzzo RP, Franchina GA, Capodanno D, et al. Selatogrel, a novel P2Y12 inhibitor: a review of the pharmacology and clinical development. Expert Opin Investig Drugs 2020;29:537-46.

6. Chen Y, Yin T, Xi S, et al. A risk score to predict postdischarge bleeding among acute coronary syndrome patients undergoing percutaneous coronary intervention: BRIC-ACS study. Catheter Cardiovasc Interv 2019;93:1194-204.

7. Martin AC, Houssany-Pissot S, Zlotnik D, et al. Management of the bleeding risk associated with antiplatelet agents. Rev Med Interne 2017;38:467-73.

8. Wiviott SD, Braunwald E, McCabe CH, et al. Prasugrel versus clopidogrel in patients with acute coronary syndromes. N Engl J Med 2007;357:2001-15.

9. Wang HY, Li Y, Xu XM, et al. Impact of Baseline Bleeding Risk on Efficacy and Safety of Ticagrelor versus Clopidogrel in Chinese Patients with Acute Coronary Syndrome Undergoing Percutaneous Coronary Intervention. Chin Med J (Engl) 2018;131:2017-24.

10. Wang DW, Sun Y, Li C, et al. P4640 Comparison of clopidogrel with ticagrelor in patients with acute coronary syndrome undergoing percutaneous coronary intervention: interim analysis of COSTIC study. European Heart Journal 2018;39. Available online: https://doi.org/10.1093/ eurheartj/ehy563.P4640

11. Feres F, Costa RA, Abizaid A, et al. Three vs twelve months of dual antiplatelet therapy after zotarolimuseluting stents: the OPTIMIZE randomized trial. JAMA 2013;310:2510-22.

12. Thygesen K, Alpert JS, Jaffe AS, et al. Fourth Universal Definition of Myocardial Infarction (2018). Circulation 2018;138:e618-51.

13. Sacco RL, Kasner SE, Broderick JP, et al. An updated definition of stroke for the 21st century: a statement for healthcare professionals from the American Heart 
Association/American Stroke Association. Stroke 2013;44:2064-89.

14. Mehran R, Rao SV, Bhatt DL, et al. Standardized bleeding definitions for cardiovascular clinical trials: a consensus report from the Bleeding Academic Research Consortium. Circulation 2011;123:2736-47.

15. De Luca L, De Servi S, Musumeci G, et al. Is ticagrelor safe in octogenarian patients with non-ST elevation acute coronary syndromes? Eur Heart J Cardiovasc Pharmacother 2018;4:12-4.

16. Gimbel M, Qaderdan K, Willemsen L, et al. Clopidogrel versus ticagrelor or prasugrel in patients aged 70 years or older with non-ST-elevation acute coronary syndrome (POPular AGE): the randomised, open-label, noninferiority trial. Lancet 2020;395:1374-81.

Cite this article as: Huang M, Li D, Li L, Wang Y, Zhang L, Chen Y, Wang DW. Factors predicting the occurrence of net adverse clinical and cerebral events in patients with acute coronary syndrome treated with clopidogrel or ticagrelor in combination with aspirin: a real-world study. Ann Transl Med 2022;10(2):98. doi: 10.21037/atm-21-7038
17. Bian L, Qiu M, Li Y, et al. Impact of extended dual antiplatelet therapy on clinical prognosis in acute coronary syndrome patients with intermediate or high ischemic risk defined by the GRACE score. Catheter Cardiovasc Interv 2020;95 Suppl 1:665-73.

18. Ge J, Li J, Yu H, et al. Hypertension Is an Independent Predictor of Multivessel Coronary Artery Disease in Young Adults with Acute Coronary Syndrome. Int J Hypertens 2018;2018:7623639.

19. Tatarunas V, Kupstyte N, Zaliunas R, et al. The impact of clinical and genetic factors on ticagrelor and clopidogrel antiplatelet therapy. Pharmacogenomics 2017;18:969-79.

(English Language Editor: C. Betlazar-Maseh) 